achieve essential national and international standards for CHD service provision on the Island. The basic concepts of such a network are of partnership, service integration and formal arrangements.

Results To date, all NI Cardiac Catheterisations are carried out in ROI. In addition, all NI emergency and urgent surgical cases have been transferred to ROI, thereby eliminating the need for children and families to travel to GB. During 2018, the Network commenced the transfer of elective surgical patients to ROI. The Network model of care includes the development of services in regional centres, the introduction of 5 Paediatrician with Expertise in Cardiology (PEC) posts across the island, implementation of an all - island CHD research strategy and the development of a joint training and education programme for health care professionals. This is the first cross-jurisdictional Clinical Network internationally and the initiatives above, complicated $\&$ often complex in a single jurisdiction, are even more so when operating cross-jurisdictionally, particularly in the midst of Brexit uncertainty.

Conclusions The all-island CHD Network is a linked groups of health professionals and organisations, working in a coordinated manner, to ensure equitable provision of high quality, clinically effective services to this complex cohort of patients. There have been many learnings, many of which are transferable to other multi-agency Networks and Systems.

\section{OC24 THE VISION AND POTENTIAL FOR A NATIONAL CHILD HEALTH E-HEALTH FRAMEWORK IN EUROPE}

\footnotetext{
1,2Michael Rigby*, 'Shalmali Deshpande, 'Grit Kuehne, 'Mitch Blair. 'Imperial College,
} London, UK; ${ }^{2}$ Keele University, Keele, UK

\subsection{6/archdischild-2019-epa.23}

Good records are vital to joined-up healthcare, ensuring delivery of preventive health, and monitoring public health. In all health spheres, digitisation and e-health are the appropriate new technologies. However, hitherto children have been badly served, and the literature shows minimal recent research in Europe.

All European countries are encouraged to lodge with WHO their current e-health plan. In 2016 the Models of Child Health Appraised (MOCHA) project examined these for 30 countries, and found only 11 (36.6\%) had specific mention of children and adolescents' issues. However, some countries had innovations in hand, including a cloud-based immunisation system in Hungary, and a parent and child portal in Latvia.

Record linkage is vital, yet only $25(83.3 \%)$ of countries have a unique health record identifier, with only $9(30 \%)$ issuing from birth. All countries but four have general use of electronic health records in child primary care. Only Croatia has a child-specific primary care EHR; most others are all age systems.

Fourteen countries have a separate child public health record system; in half of these are appointments issued. Countries vary in data exchange policies between primary and secondary care; with community and preventive health providers, and with the school health service. There is also variation between countries on children's record access, and on confidentiality from parents.

Standards bodies are involved in a European Patient Summary, which only marginally impacts on children's needs. ECDC promotes all-age Immunisation Information Systems.
WHO promotes Home Based Records, but there are no standards, or established best practice.

Web sites and mobile phone apps are modern health support tools, but they can be unreliable and can breach privacy. Only six countries have accreditation processes for apps, and eight have them for web sites. A few countries have instigated specific web sites for health advice for children.

E-health is an ideal way to facilitate child health clinicians and give children relevant easy access to services. Several good initiatives exist, and ECDC and standards bodies are contributing, but overall the situation is poor. But with evaluation and collaboration involving professional and standards bodies, WHO and the EU, existing best practice could be drawn on to identify an optimal model on which all countries could draw.This would include:

Record linkage from birth

Standards for data items and functionality including condition-specific algorithms.

Access, sharing, and consent protocols

Approved anonymised use for research

Web site and app approval criteria.

\section{OC25 GROWTH AND NUTRITION SUPPORT IN INFANTS WITH UNIVENTRICULAR PHYSIOLOGY}

${ }^{1}$ Anne Marie Shine*, ${ }^{1}$ Leah Foyle, ${ }^{1}$ Aoife 0 Neill, ${ }^{2}$ Colin J McMahon. ${ }^{1}$ Department of Clinical Nutrition and Dietetics, Our Lady's Childrens Hospital, Crumlin., Dublin 12, Ireland; 2Department of Paediatric Cardiology, Our Lady's Childrens Hospital, Crumlin., Dublin 12, Ireland

\subsection{6/archdischild-2019-epa.24}

Background Growth impairment in infants with univentricular congenital heart disease is well documented. Meeting nutritional requirements in the early phase of infancy can be particularly challenging as these infants undergo complex surgical palliation within the first few days of life. This audit is a retrospective study aiming to evaluate the nutritional status of these infants.

Method Eighty-nine infants with single ventricle physiology underwent a surgical Norwood or a hybrid/interventional procedure (stage 1) as a neonate in OLCHC during the time period January 2014 - December 2018. Subject data collected included demographics, procedure type, anthropometry, mode and type of feeding, nutritional intake and nutrition related complications. Weight for Age Z scores (WAZ) were calculated using the World Health Organisation Standards.

Results WAZ $<-2$ is a screening criterion for undernutrition. Mean WAZ at birth was -0.1 on discharge was -1.44 and prior to stage 2 surgery Bidirectional Glenn was -1.26 . On discharge (median length of stay 25 days) post stage 1 procedure, 29\% of infants had a mean WAZ $<-2$. A paired sample $\mathrm{T}$ test demonstrated that the drop in mean WAZ from birth to discharge is statistically significant $\mathrm{p}$ value $<0.001$ (significance $<0.05$ ).

Median number of days to any form of nutrition support was 3 and to first enteral feed was 6. Median number of days to achieve basic energy requirements from $\mathrm{EN}$ was 16. Preoperative trophic feeds and parenteral nutrition were provided to $18 \%$ and $38 \%$ of infants respectively. Vocal chord palsy and chylothorax arose frequently amongst the Norwood group with one in five infants experiencing such difficulties. On discharge post stage 1 intervention, $48 \%$ of infants were 
receiving tube feeds. Mean daily calorie intake on week 1 and week 2 was $24 \mathrm{kcal} / \mathrm{kg} /$ day and $59 \mathrm{kcal} / \mathrm{kg} /$ day respectively.

Conclusions Reaching nutrition goals is often complicated and delayed in the neonatal pre and post-operative period. This is evident here with delays in provision of nutrition support and in time to reach basic energy requirements. Our results concur with the literature with the greatest decline in WAZ occurring in the neonatal period. During a critical period of growth and development infants with HLHS experience significant challenges and nutritional compromise. It is necessary to develop strategies and guidelines directed at improving the nutritional intake and status of this vulnerable group.

\section{OC26 FEATURES OF GUT MICROBIOTA COMPOSITION IN OBESE ADOLESCENTS}

'Lyubov Rychkova, ${ }^{2}$ Evgenia Novikova*, ${ }^{2,3}$ Natalia Belkova, ${ }^{2}$ Anna Pogodina, ${ }^{2}$ Anastasiya Romanitsa, ${ }^{3,4}$ Sergey Feranchuk. ${ }^{1}$ Scientific Centre of Family Health and Human Reproduction Problems, Irkutsk, Russian Federation; ${ }^{2}$ Scientific Centre for Family Health and Human Reproduction Problems, Irkutsk, Russian Federation; ${ }^{3}$ Limnological Institute SB RAS, Irkutsk, Russian Federation; ${ }^{4}$ National Research Technical University, Irkutsk, Russian Federation

\subsection{6/archdischild-2019-epa.25}

Introduction Obesity is a multifactorial disease, which may be caused by genetic, psychological causes, obesogenic lifestyle including disturbance in energy balance and a sedentary lifestyle. Gut microbiota takes part in digestion of plant polysaccharides, and also it produces a strong influence on lipid and cholesterol metabolism, therefore gut microbiota dysbiosis may leads to metabolic dysfunction including obesity. Therefore, we made an aim to detect dominant types of gut microbiota at patients with obesity and normal weight and evaluate biochemical parameters of serum alanine transaminase (ALT) and serum alkaline phosphatase (AP), which are obesity-associated metabolic risk factors.

Methods The total participant's number was 40. 18 subjects were obese adolescents $\left(B M I=32,57 \pm 4,29 \mathrm{~kg} / \mathrm{m}^{2}\right)$, the 22 subjects were healthy adolescents $\left(B M I=20,01 \pm 1,66 \mathrm{~kg} / \mathrm{m}^{2}\right)$. These two groups were comparable by ethnicity (all participants are Caucasians), gender (10 males and 8 females at the obese group; 13 males and 9 females at the control group), and age (mean age was $14.75 \pm 1.52$ yrs for obese normal weight and $14.73 \pm 1.55$ yrs for the normal weight group).

Metagenome sequencing of V3-V4 variable regions of $16 \mathrm{~S}$ rDNA were done by Novogene Company (China). Concentration of serum ALT and AP were measured using Mindray Automatic Biochemistry Analyzer. Data were analyzed using the bioinformatics services bri-shur.com. For group comparison the $t$-test was used. Statistical significance was accepted at the $\mathrm{p} \leq 0.05$ level.

Results Assessment of OTU in two groups of adolescents healthy and obesity - revealed the minimal values in the obesity group, the maximal - in the healthy, which showed the decrease in gut microbiome diversity in obesity. There was identified significant difference between gut microbiota composition at the obesity and control groups: the Dorea genus (phylum Firmicutes) dominated at obese patients $(p=0.05)$. Otherwise the groups weren 't significantly different from each other, but there was a tendency of Bacteroides, Parabacteroides (the phylum Bacteroidetes) and Slackia, Collinsella (the phylum Actinobacteria) prevalence at the obesity group (the $\mathrm{p} \geq 0.05$ ).
Obese adolescents had the mean ALT as $25,34 \pm 13,44$, and the mean AP was $143,63 \pm 83,69$, but this is not statistically reliable $(\mathrm{p}>0,05)$. The mean ALT of adolescents without obesity was $18,87 \pm 13,26$, and the mean AP was 165,76 199 , $(\mathrm{p}>0,05)$.

Conclusion We report that obese adolescents had higher levêts of ALT and lower levels of AP in their serum, and less divese gut microbiome communities with higher relative abundan of the main bacteria phyla like Firmicutes, Bacteroidet Actinobacteria.

\section{OC27 NATIONAL NEWBORN SCREENING FOR CYSTIC FIBROSIS: GENETIC DATA FROM THE FIRST 6 YEARS}

${ }^{1}$ Erina Sasaki*, ${ }^{2}$ Marija Kostocenko, ${ }^{1}$ Niamh Lang, ${ }^{1}$ Tara Clarke, ${ }^{1}$ Melissa Rogej ${ }^{1}$ Rebecca Muldowney, ${ }^{1}$ Alana Ward, 'David Barton, 'Sally Ann Lynch. 'Departmentüg Clinical Genetics, Our Lady's Children's Hospital, Dublin, Ireland; 'School of Medicine, Robal College of Surgeons in Ireland, Dublin, Ireland

10.1136/archdischild-2019-epa.26

Background Cystic Fibrosis (CF) is the most common lite threatening autosomal recessive multisystem disease in the Republic of Ireland (ROI); with a previously quoted incidenfe of 1 in 1353 and carrier rate of 1 in 19 . Screening for ofF was incorporated in the National new born screening (NBS) programme in July 2011 in the ROI. The benefit of eady diagnosis is well documented. The purpose of the screening to identify classic CF cases to allow early diagnosis and inte vention and improve prognosis. A cut off point of the t品 1\% Immunoreactive Trypsinogen (IRT) was taken as an indi⿸厃㔾 tion for 38 mutation panel molecular screening in the R National screening programme to maximise identification $\overrightarrow{\emptyset \mathrm{f}}$ affected typical CF cases and to minimise detection $8 \mathrm{f}$ carriers.

Methods All neonates from July 2011 to Dec 2017 who h⿳亠丷厂巾d an elevated IRT on NBS were tested with 38 CFTR ge若 mutation panel and included in this study. Data from clini a्al and laboratory database were analysed and cross-referencö. with patient charts. The Non-NBS database was used to track down cascade relatives.

Results In the first 6 and a half years a total of 5,094 ne borns $(1.16 \%$ of total births in the period) were screened CFTR mutation. During this period, $170 \mathrm{CF}$ affected cases, 22 CFSPID (CF screening positive, Inconclusive diagnosis) a de 325 healthy carriers were identified. Phe508del was the most common mutation (75\%) followed by Gly551Asp (9.4\%). (56\%) were homozygous for Phe508del, 17 (10\%) were co吕pound heterozygous for Phe508del and Gly551Asp and $(13 \%)$ were compound heterozygous for Gly551Asp. Hene्e, $69 \%$ of identified as an affected new-borns are eligible to Nox orphan drugs. There was one missed diagnosis.

Conclusion The National new-born screening programme toss been successful with only 1 missed diagnosis and less them $50 \%$ of carriers identified than predicted. We identified twe the number of affected new-born carrying the Gly551A mutation than previously quoted figures from elsewhere :in Western Europe. As these are eligible for new orphan drut this is important for drug reimbursement nationally. T满e revised incidence of $\mathrm{CF}$ in the ROI is less than previoक reports (likely due to net immigration). We estimate the no revised incidence to be 1 in 2570 and the carrier frequency 1 in 25 . 